\title{
ON A CLASS OF THIRD ORDER NEUTRAL DELAY DIFFERENTIAL EQUATIONS WITH PIECEWISE CONSTANT ARGUMENT
}

\section{GARYFALOS PAPASCHINOPOULOS}

Democritus University of Thrace

School of Engineering

67100 Xanthi, Greece

(Received October 26, 1990 and in revised form May 15, 1991)

ABSTRACT. In this paper we study existence, uniqueness and asymptotic stability of the solutions of a class of third order neutral delay differential equations with piecewise constant argument.

KEY WORDS AND PHRASES. Neutral delay differential equations with piecewise constant argument, difference equation, existence and uniqueness of solutions, asymptotic stability.

1980 AMS SUBJECT CLASSIFICATION CODE. 34K05, $34 \mathrm{~K} 15$.

\section{INTRODUCTION}

Recently there has been an increase in interest in the study of differential equations with piecewise constant argument. See Aftabizadeh, Wiener and Xu [1], Cooke and wiener [2], [3], Huang [4], Ladas, Partheniadis and Schinas [5], Partheniadis [6].

In this paper we study the third order neutral delay differential equations with piecewise constant argument of the form

$$
\frac{d^{3}}{d t^{3}}(y(t)+p y(t-1))=-q y([t-1])
$$

where $t e[0, \infty), \quad p, q$ are real constants and $[\cdot]$ denotes the greatest-integer function. It is worthwile to study equations of the form (1) because they include both constant and piecewise constant delays.

A function $y:[-1, \infty) \rightarrow R$ is a solution of (1) if the following conditions are satisfied:

(i) $y$ is continuous on the set $[-1, \infty)$,

(ii) $\frac{d^{2}}{d t^{2}}(y(t)+p y(t-1))=g(t)$ exists on $[0, \infty)$ and $g$ is continuous on $[0, \infty)$, (iii) $\frac{d^{3}}{d t^{3}}(y(t)+p y(t-1))$ exists on $[0, \infty)$ except possibly at the points $t=n$, $n \in\{0,1, \ldots\}$ where one-sided third derivatives exist,

(iv) $y$ satisfies (1) on each interval $[n, n+1)$, ne $\{0,1, \ldots\}$.

In Proposition 1 of this paper we prove that for every initial function $y_{0}:[-1,0] \rightarrow R$ continuous on $[-1,0]$ and for every $a_{1}, a_{2}$ eR there exists a unique solu- 
tion $y(t)$ of (1) such that $y(t)=y_{0}(t), \quad t e[-1,0], y(1)=a_{1}$ and $y(2)=a_{2}$. We prove also that equation (1) is not asymptotically stable (see Proposition 2 below).

We note that similar results for the first and the second order differential equations of the form (1) are included in [6].

2. MAIN RESULTS.

We prove now our main results.

PROPOSITION 1. Equation (1) has a unique solution $y(t)$ such that

$$
y(t)=\left\{\begin{array}{lll}
y_{0}(t) & t \in[-1,0] & \text { for } p \neq 0 \\
a_{-1} & t=-1 & \text { for } p=0 \\
a_{0} & t=0 & \text { for } p=0 \\
a_{1} & t=1 & \\
a_{2} & t=2 &
\end{array}\right.
$$

where $y_{0}:[-1,0] \rightarrow R$ is a continuous function on $[-1,0], a_{-1}, a_{0}, a_{1}, a_{2}$ are real constants and $a_{0}=y_{0}(0), a_{-1}=y_{0}(-1)$ if $p \neq 0$.

The solution $y(t)$ is defined by

$$
\begin{aligned}
& y(t)=(-p)^{n+1} y_{o}(\theta-1)+\sum_{k=0}^{n}(-p)^{n-k}\left(\frac{\left(\theta^{2}-\theta\right)}{2} a_{k+2}+\right. \\
& +\left(\frac{\theta^{2}(p-2)}{2}+\frac{\theta(4-p)}{2}\right) a_{k+1}+\left(\frac{\theta^{2}}{2}\left(\frac{q}{6}-2 p+1\right)+\theta\left(2 p-\frac{q}{12}-\frac{3}{2}\right)+1\right) a_{k} \\
& \left.+\left(-\frac{q}{6} \theta^{3}+\frac{\theta^{2}}{2}\left(p+\frac{5 q}{6}\right)-\frac{\theta}{2}\left(3 p+\frac{q}{2}\right)+p\right) a_{k-1}\right)
\end{aligned}
$$

where $a_{n}$ satisfies the difference equation

$$
a_{n+4}+(p-3) a_{n+3}+\left(\frac{q}{6}-3 p+3\right) a_{n+2}+\left(\frac{2 q}{3}+3 p-1\right) a_{n+1}+\left(\frac{q}{6}-p\right) a_{n}=0 .
$$

PROOF. Consider a solution $y(t)$ of (1) which satisfies (2). For each $t \in[-1, \infty)$ there exists a $n \in\{-1,0, \ldots\}$ such that $n \leqq t<n+1$. We set

$$
y(n)=a_{n}, \quad n \in\{-1,0, \ldots\} \text {. }
$$

Then from (1) for $t \geqq 0, t \in[n, n+1), \quad n \in\{0,1, \ldots\}$ it holds

$$
\frac{d^{3}}{d t^{3}}(y(t)+p y(t-1))=-q a_{n-1} \text {. }
$$

If

$$
\beta_{n}=\frac{d^{2}}{d t^{2}}(y(t)+p y(t-1)) \text { at } t=n, \quad n \in\{0,1, \ldots\}
$$

then by integrating (6) from $n$ to $t \in[n, n+1), n \in\{0,1, \ldots\}$ we take

$$
\frac{d^{2}}{d t^{2}}(y(t)+p y(t-1))=\beta_{n}-q(t-n) a_{n-1} .
$$

Moreover if

$$
c_{n}=\frac{d}{d t}(y(t)+p y(t-1)) \text { at } t=n, \quad n e\{0,1, \ldots\}
$$


by integrating (8) from $n$ to $t, t e[n, n+1), n \in\{0,1, \ldots\}$ we have

$$
\frac{d}{d t}(y(t)+p y(t-1))=c_{n}+(t-n) \beta_{n}-\frac{g}{2}(t-n)^{2} a_{n-1} .
$$

Finally by integrating (10) from $n$ to $t, t \in[n, n+1), n \in\{0,1, \ldots\}$ and using we obtain

$$
y(t)+p y(t-1)=a_{n}+p a_{n-1}+(t-n) c_{n}+\frac{(t-n)^{2}}{2} \beta_{n}-\frac{q}{6}(t-n)^{3} a_{n-1} .
$$

Applying now Lemma $3[6, p .96]$ to (11) we get

$$
y(t)=(-p)^{n+1} y_{0}(\theta-1)+\sum_{k=0}^{n}(-p)^{n-k}\left(a_{k}+p a_{k-1}+\theta c_{k}+\frac{\theta^{2}}{2} \beta_{k}-\frac{q}{6} \theta^{3} a_{k-1}\right)
$$

where $t=n+\theta, \quad 0 \leqq \theta \leqq 1, \quad n \in\{0,1, \ldots\}$.

Since $y(t)$ is continuous in $[-1, \infty)$ by taking the limit as $t \rightarrow n+1$ in (11) and using (5) we get for $n \in\{0,1, \ldots\}$

$$
a_{n+1}+p a_{n}=a_{n}+\left(p-\frac{q}{6}\right) a_{n-1}+c_{n}+\frac{1}{2} \beta_{n} .
$$

If we take the limit as $t \rightarrow n+1$ in (8) and since the function $g(t)=\frac{d^{2}}{d t^{2}}(y(t)+$ $+p y(t-1))$ is continuous, using (7) we have for $n \in\{0,1, \ldots\}$

$$
\beta_{n+1}=\beta_{n}-q a_{n-1} \text {. }
$$

Obviously the function $h(t)=\frac{d}{d t}(y(t)+p y(t-1))$ is continuous on $[0, \infty)$. Then If we take the limit as $t \rightarrow n+1$ in (10) and using (9) we obtain for $n \in\{0,1, \ldots\}$

$$
c_{n}=c_{n+1}-\beta_{n}+\frac{q}{2} a_{n-1} \text {. }
$$

By eliminating $b_{n}, c_{n}$ from (13), (14), (15) we can easily get

$$
-\frac{\beta_{n+1}}{2}+c_{n+1}=a_{n+1}+(p-1) a_{n}+\left(\frac{q}{6}-p\right) a_{n-1} .
$$

If we eliminate $b_{n+1}, c_{n+1}$ from $(14),(16)$ and the relation which is derived from (13) by setting $n+1$ instead of $n$, then it holds

$$
\beta_{n}=a_{n+2}+(p-2) a_{n+1}+\left(\frac{q}{6}-2 p+1\right) a_{n}+\left(p+\frac{5 q}{6}\right) a_{n-1} .
$$

Using (13) and (17) for $n \in\{0,1, \ldots\}$ we can easily take

$$
c_{n}=-\frac{1}{2} a_{n+2}+\left(2-\frac{p}{2}\right) a_{n+1}+\left(2 p-\frac{q}{12}-\frac{3}{2}\right) a_{n}-\left(\frac{3 p}{2}+\frac{q}{4}\right) a_{n-1} .
$$

Therefore from (12), (17) and (18) we can easily show that $y(t)$ satisfies (3). Now from relations (14) and (17) it is easy to prove that a satisfies the difference equation (4).

Obviously if $p \neq \frac{q}{6}$ there exists a unique solution $a_{n}$ of (4) with initial values $a_{-1}, a_{0}, a_{1}, a_{2}$ and if $p=\frac{q}{6}, p \neq \frac{1}{7}, q \neq \frac{6}{7}$ (resp. $p=\frac{1}{7}, \quad q=\frac{6}{7}$ ) there exists a unique solution of (4) with initial values $a_{0}, a_{1}, a_{2}$ (resp. $a_{1}$, $\left.a_{2}\right)$. Therefore we proved that if $y(t)$ is a solution of (1) which satisfies then is defined by (3) and (4) and is uniquely determined by $y_{0}, a_{1}, a_{2}$ if $p \neq 0$ 
and $a_{-1}, a_{0}, a_{1}, a_{2}$ if $p=0$.

Conversely we can easily show that the function $y(t)$ defined by ( 3 ) and (4) is a solution of (1) which satisfies (2). Thus the proof of the proposition is completed.

Using (3) and the same argument as in the proof of the Corollary 4 [6,p.110-111] we can easily prove the following corollary.

COROLLARY 1. Let $y(t)$ be the solution of (1) which satisfies (2). Then it holds

$$
\begin{aligned}
y(t) & =(-p)^{n+1} y_{0}(\theta-1)+(-p)^{n+1}\left(-\frac{\theta^{2}}{2}\left(1+\frac{q}{6}\right)+\frac{\theta}{2}\left(\frac{q}{6}+3\right)-1\right) a_{-1}+ \\
& +(-p)^{n+1}\left(\theta^{2}(1-p)+\theta(p-2)\right) a_{0}+(-p)^{n+1} \frac{\left(-\theta^{2}+\theta\right)}{2} a_{1}+ \\
& +\left(\frac{\theta^{2}}{2}\left(\frac{q}{6}+1\right)-\frac{\theta}{2}\left(\frac{q}{6}+3\right)+1\right) a_{n}+\left(2 \theta-\theta^{2}\right) a_{n+1}+\frac{\left(\theta^{2}-\theta\right)}{2} a_{n+2}+ \\
& +\sum_{k=0}^{n}(-p)^{n-k}\left(-\frac{q}{6} \theta^{3}+\frac{\theta^{2} q}{12}(5-p)+\frac{\theta q}{4}\left(\frac{p}{3}-1\right)\right) a_{k-1}
\end{aligned}
$$

where $t=n+\theta, \quad 0 \leq \theta \leq 1, \quad n \in\{0,1, \ldots\}$.

In Proposition 2 of this paper we prove that (1) is not asymptotically stable. We need the following lemma.

LEMMA 1. Consider the difference equation

$$
a_{n+4}+\mu_{1} a_{n+3}+\mu_{2} a_{n+2}+\mu_{3} a_{n+1}+\mu_{4} a_{n}=0
$$

where $n \in\{-1,0,1, \ldots\}$ and $\mu_{i}, 1=1,2,3,4$ are real constants. Then (19) is asymptotically stable if and only if the following conditions are satisfied:

$$
\begin{aligned}
& \text { (i) } \lambda_{1} \neq 0, \quad \text { (ii) } \frac{\lambda_{2}}{\lambda_{1}}>0, \quad \text { (iii) } \frac{\lambda_{3}}{\lambda_{1}}>0, \quad \text { (iv) } \frac{\lambda_{5}}{\lambda_{1}}>0 \\
& \text { (v) } \frac{\lambda_{4}}{\lambda_{1}}\left(\frac{\lambda_{2} \lambda_{3}}{\lambda_{1}^{2}}-\frac{\lambda_{4}}{\lambda_{1}}\right)>\frac{\lambda_{2}^{2} \cdot \lambda_{5}}{\lambda_{1}^{3}}
\end{aligned}
$$

where

$$
\begin{aligned}
& \lambda_{1}=1+\mu_{1}+\mu_{2}+\mu_{3}+\mu_{4}, \quad \lambda_{2}=4+2 \mu_{1}-2 \mu_{3}-4 \mu_{4}, \\
& \lambda_{3}=6-2 \mu_{2}+6 \mu_{4}, \lambda_{4}=4-2 \mu_{1}+2 \mu_{3}-4 \mu_{4}, \quad \lambda_{5}=1-\mu_{1}+\mu_{2}-\mu_{3}+\mu_{4} .
\end{aligned}
$$

PROOF. It is known that (19) is asymptotically stable if and only if every root $v$ of the characteristic equation

$$
v^{4}+\mu_{1} v^{3}+\mu_{2} v^{2}+\mu_{3} v+\mu_{4}=0
$$

satisfies $|v|<1$. Then it is clear that $\lambda_{1} \neq 0$. Therefore using the Mobius transformation

$$
v=\frac{z+1}{z-1}
$$


to (22) we can take the equation

$$
z^{4}+\frac{\lambda_{2}}{\lambda_{1}} z^{3}+\frac{\lambda_{3}}{\lambda_{1}} z^{2}+\frac{\lambda_{4}}{\lambda_{1}} z+\frac{\lambda_{5}}{\lambda_{1}}=0
$$

where the constants $\lambda_{i}, i=1,2,3,4,5$ are defined in (21).

It is easy to show that every root $v$ of (22) satisfies $|v|<1$ if and only if $\operatorname{Rez}<0$, where $\operatorname{Rez}$ is the real part of $z$ and $z$ is the root of (23). Using Routh-Hurwitz criteria [7,p.158] we have Rez $<0$ if and only if all the conditions (20) are satisfied. This completes the proof of the lemma.

PROPOSITION 2. Equation (1) is not asymptotically stable.

PROOF. Suppose that (1) is asymptotically stable. Then the difference equation (4) is also asymptotically stable. We apply Lemma 1 to (4). It is easy to show that

$$
\lambda_{1}=q \text { and } \lambda_{2}=-2 q \text {. }
$$

Then $\frac{\lambda_{2}}{\lambda_{1}}=-2<0$ which contradicts the second condition of (20). Therefore is not asymptotically stable.

ACKNOWLEDGMENT. I would like to thank the referee for his valuable suggestions which led to the presentation of this paper.

\section{REFERENCES}

1. AFTABIZADEH, A.R., WIENER, J. and XU, JiM. Oscillatory and periodic solutions of delay differential equations with piecewise constant argument, Proc. Amer. Math. Soc. 99 (1987), 673-679.

2. COOKE, K.L. and WIENER, J. Retarded differential equations with piecewise constant delays, J.Math. Anal. App1. 99 (1984), 265-297.

3. COOKE, K.L. and WIENER, J. An equation alternately of retarded and advanced type, Proc. Amer. Math. Soc. 99 (1987), 726-732.

4. HUANG, Y.K. Oscillations and asymptotic stability of solutions of first order neutral differential equations with piecewise constant argument, J. Math. Ana1. App1. 149 (1990), 70-85.

5. LADAS, G., PARTHENIADIS, E.C. and SCHINAS, J. Existence theorems for second order differential equations with piecewise constant argument, Differential Equations, (Xanthi, 1987), 389-395, Lecture Notes in Pure and Appl. Math., 118, Dekker, New York, 1989.

6. PARTHENIADIS, E.C. Oscillations and asymptotic behavior of solutions of delay and neutral delay differential equations, Ph.D. Thesis, University of Rhode Island (1988).

7. COPPEL, W.A. Stability and asymptotic behavior of differential Heath. Boston, 1965. 


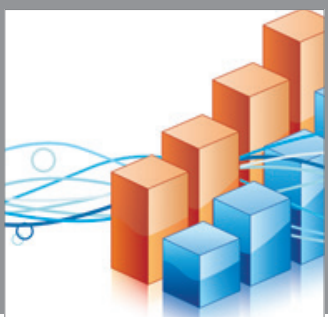

Advances in

Operations Research

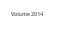

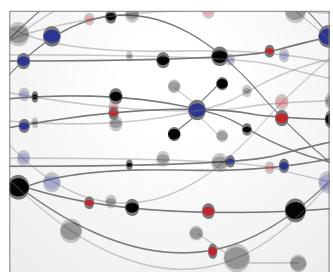

\section{The Scientific} World Journal
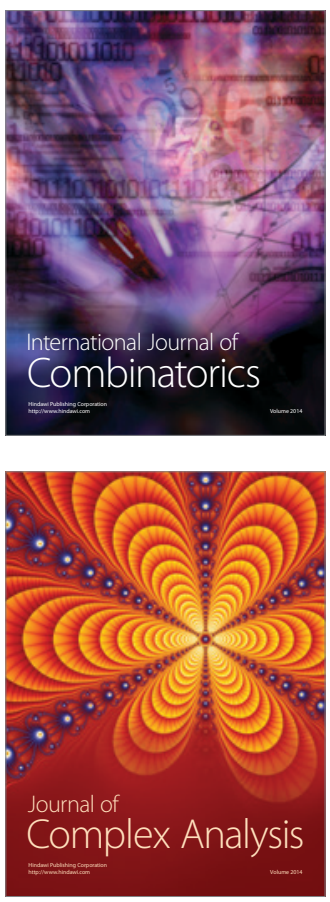

International Journal of

Mathematics and

Mathematical

Sciences
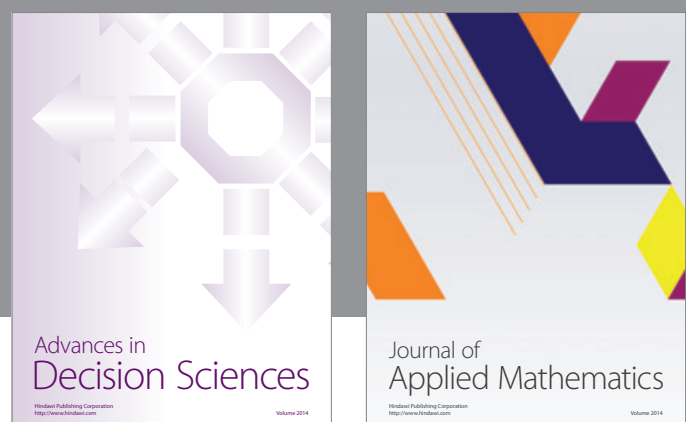

Journal of

Applied Mathematics
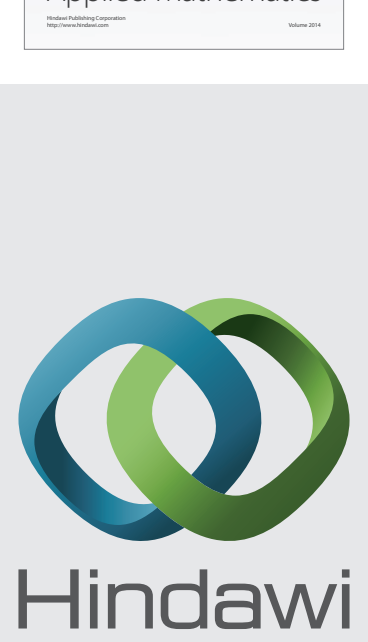

Submit your manuscripts at http://www.hindawi.com
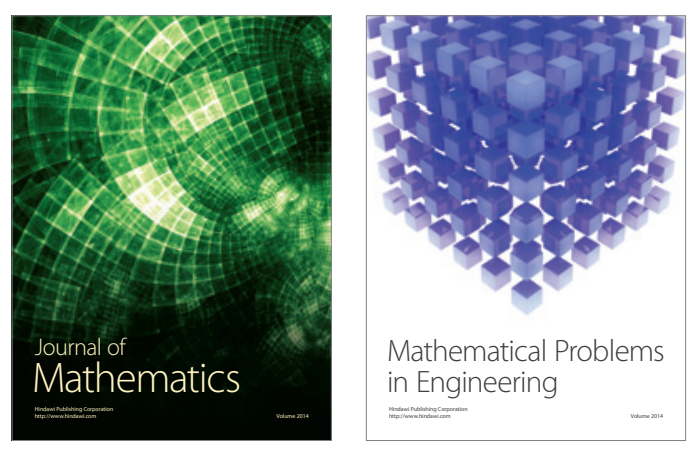

Mathematical Problems in Engineering
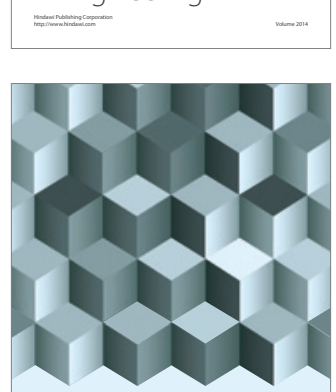

Journal of

Function Spaces
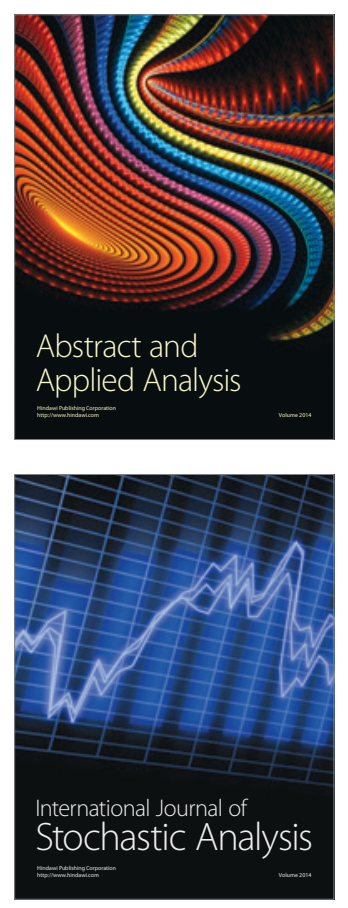

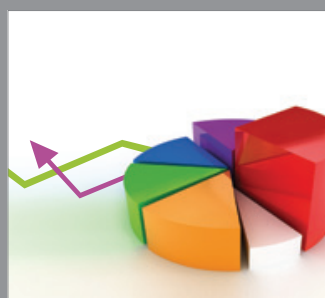

ournal of

Probability and Statistics

Promensencen
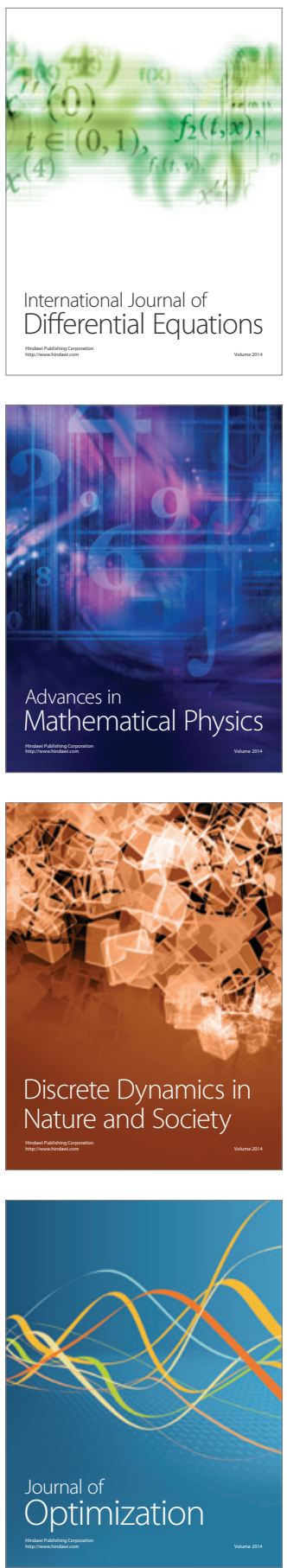